\title{
DISTRIBUTED EYE BLINK ARTIFACT REMOVAL IN A WIRELESS EEG SENSOR NETWORK
}

\author{
Alexander Bertrand*, Marc Moonen \\ KU Leuven, Dept. Electrical Engineering ESAT \\ Stadius Center for Dynamical Systems, Signal Processing and Data Analytics \\ Kasteelpark Arenberg 10, B-3001 Leuven, Belgium \\ E-mail: alexander.bertrand@esat.kuleuven.be; marc.moonen@esat.kuleuven.be
}

\begin{abstract}
In this paper, we present a distributed algorithm to remove eye blink artifacts from electroencephalography (EEG) signals recorded in a modular high-density EEG system, referred to as a wireless EEG sensor network (WESN). A WESN is a particular instance of a wireless body area network for long-term non-invasive neuromonitoring, which is amenable to extreme miniaturization and low-power system design. We first propose a centralized algorithm for eye blink artifact removal (EBAR) based on the multi-channel Wiener filter (MWF). We then show how this MWF-based EBAR algorithm can be implemented in a distributed fashion to remove the eye blink artifacts in each EEG node without centralizing all the raw EEG data. Instead, the EEG nodes share fused EEG signals with each other. Nevertheless, it can be shown that the estimation performance of the distributed algorithm is equivalent to the performance of the centralized MWF, as if each EEG node had access to all the EEG channels of the WESN. This is also experimentally validated by means of recorded EEG data.
\end{abstract}

Index Terms - Wireless body area networks, EEG, distributed signal processing, distributed estimation.

\section{INTRODUCTION}

Electroencephalography (EEG) is a non-invasive neuromonitoring technique to record biopotential signals generated by the brain. For many applications, there is a growing interest in chronic or long-term high-density EEG monitoring during the everyday life of a subject (over multiple days, months, or even years, and with up to 60 or more EEG channels) [1-6]. However, the development of a system for such long-term high-density EEG monitoring requires significant technological advances, in particular to make it wireless, low-power, real-time, and amenable to extreme miniaturization.

To facilitate these features, there is currently an ongoing evolution towards modular EEG systems, where separate miniature EEG

\footnotetext{
${ }^{*}$ The work of A. Bertrand was supported by a Postdoctoral Fellowship of the Research Foundation - Flanders (FWO). This work was carried out at the ESAT Laboratory of KU Leuven, in the frame of KU Leuven Research Council CoE EF/05/006 'Optimization in Engineering' (OPTEC) and PFV/10/002 (OPTEC), Concerted Research Action GOA-MaNet, the Belgian Programme on Interuniversity Attraction Poles initiated by the Belgian Federal Science Policy Office IUAP P7/23 (BESTCOM, 20122017) and IUAP P7/19 (DYSCO, 2012-2017), Research Project FWO nr. G.0763.12 'Wireless acoustic sensor networks for extended auditory communication' and project HANDiCAMS. The project HANDiCAMS acknowledges the financial support of the Future and Emerging Technologies (FET) programme within the Seventh Framework Programme for Research of the European Commission, under FET-Open grant number: 323944. The scientific responsibility is assumed by its authors.
}

nodes are deployed on the scalp $[4,7,8]$, replacing the bulky headsets that are used nowadays. Each EEG node is then equipped with one or more electrodes, a local processing unit and a wireless radio to transmit its data to a base station and/or to other EEG nodes. We refer to such a modular EEG system as a wireless EEG sensor network (WESN), which is a particular instance of a wireless body area network $[9,10]$. It is believed that these WESNs will be a key enabler for long-term high-density EEG monitoring. Furthermore, the modularity of the system also allows for a more flexible deployment (in terms of required number of nodes, as well as the addition/removal/replacement of nodes).

Several advances towards the development of such WESNs have been made in terms of hardware design $[1,4,6]$, wireless communication link design $[7,8]$, and the design of dry and separately deployable (self-attaching) EEG electrodes [4,11-13]. However, it appears that the signal processing (SP) aspects have not yet received much attention. One important challenge is to design SP algorithms that fully exploit the per-node processing capabilities to reduce the communication cost, as the wireless communication between the nodes typically dominates the energy consumption [1]. Onboard preprocessing of EEG signals has been briefly explored in $[1,2,14,15]$, where each EEG channel is processed independently. However, EEG processing often relies on multi-channel algorithms which exploit the correlation between the EEG signals recorded at different electrodes. For example, the removal of eye blink artifacts, which are one of the most pronounced artifacts in EEG signals, indeed requires multi-channel algorithms, such as independent component analysis (ICA) [16, 17], canonical correlation analysis [18], or other spatial filtering methods [19]. If these algorithms are to exploit the full correlation structure of the EEG grid, in principle all the raw data has to be centralized, which hampers the use of the per-node processing capabilities to reduce the communication cost and to distribute the computational cost ${ }^{1}$.

In this paper, we present a distributed multi-channel algorithm for the removal of eye blink artifacts, which facilitates per-node processing, and indeed exploits the full correlation structure of the EEG grid without centralizing the raw EEG signals. Instead, the EEG nodes share fused/compressed signals among each other or with a base station, and each node contributes to the computational task. To this end, we first describe an eye blink artifact removal (EBAR) algorithm based on the multi-channel Wiener filter (MWF), which has originally been proposed for speech enhancement in microphone arrays [20], but which has more recently also been successfully applied in EEG signal processing [21,22]. We propose this MWF-based

\footnotetext{
${ }^{1}$ The computational complexity of most spatial filtering techniques typically scales quadratically or cubically in the number of input signals.
} 


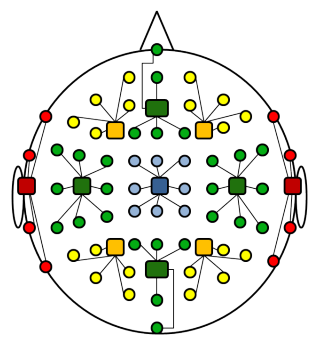

(a) Multi-electrode nodes

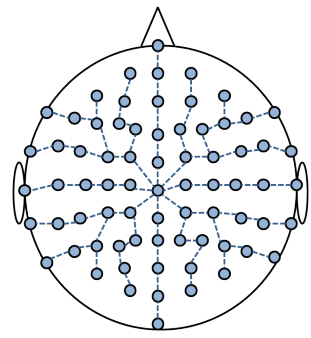

(b) Single-electrode nodes in a tree topology
Fig. 1. A 75-electrode WESN in two different topologies with electrode placement based on an extension of the standard 10-20 system.

method because it is amenable to a distributed implementation using the so-called distributed adaptive node-specific signal estimation (DANSE) algorithm [23,24]. It can be shown that the DANSE algorithm estimates the eye blink artifacts at each node by optimally exploiting the full correlation structure as if each node had access to all the EEG channels, i.e., equivalent to the centralized MWF. This theoretical statement is validated by means of recorded EEG data, where we also compare the performance of the proposed distributed EBAR algorithm with a commonly used centralized ICAbased EBAR algorithm.

\section{PROBLEM STATEMENT AND NOTATION}

In this paper, we assume that each EEG node of the WESN has access to multiple EEG channels, which is the case if, e.g., each node is equipped with multiple electrodes (see Fig. 1(a)), or if the WESN is organized in a hierarchical topology in which master nodes collect signals from multiple EEG nodes in their neighborhood. We also assume that the WESN is fully connected, i.e., a signal broadcast by one node can be observed by all the other nodes in the network. Note that this fully-connected topology also automatically appears if each node transmits its signal(s) to a base station, such as a smartphone, since this long-range transmission allows the other nodes to also observe the broadcast signal. However, it is noted that the DANSE algorithm described in Section 4 can also be extended to multi-hop topologies with in-network signal fusion [25] (see, e.g., Fig. 1(b)), but this is beyond the scope of this paper. The different nodes can use a common reference electrode ${ }^{2}$, or each node can use one of its local electrodes as a reference. It is noted that this choice is irrelevant in the assumed data model or algorithm design, and we will therefore make abstraction of this in the sequel.

The set of nodes is denoted as $\mathcal{K}=\{1, \ldots, K\}$, where $K$ is the total number of nodes in the WESN. The vector $\mathbf{y}_{k}[t]$ represents an $M_{k}$-channel signal in the discrete time index $t$, corresponding to the $M_{k}$ EEG channels that are collected by node $k$, and $y_{k, j}[t]$ refers to the $j$-th EEG channel at node $k$. The discrete time index $t$ will be omitted in the sequel for conciseness. We denote $\mathbf{y}=\left[\mathbf{y}_{1}^{T} \ldots \mathbf{y}_{K}^{T}\right]^{T}$ as the $M$-channel signal in which all the $\mathbf{y}_{k}$ 's, $\forall k \in \mathcal{K}$, are stacked, and where $M=\sum_{k \in \mathcal{K}} M_{k}$ represents the total number of EEG channels in the WESN (we use $y_{j}$ to refer to the $j$-th channel of $\mathbf{y}$ ). The EEG signals in $\mathbf{y}$ consist of two (hidden) signal components $\mathbf{d}$

\footnotetext{
${ }^{2}$ This would require spanning a small wire between the nodes in a daisy chain or using a head cap of conductive fabric, as proposed in [4] (note that this is only used for the common reference voltage, i.e., data transmission or sharing still happens over wireless communication links).
}

and $\mathbf{v}$, i.e.,

$$
\mathbf{y}=\mathbf{d}+\mathbf{v}
$$

where $\mathbf{d}$ is an $M$-channel signal containing the eye blink artifacts in each EEG channel, and $\mathbf{v}$ is the $M$-channel signal containing the artifact-free EEG signals. The goal is to first estimate the signal $\mathbf{d}$, i.e., the eye blink artifacts in each EEG channel, and then subtract it from $\mathbf{y}$ to estimate $\mathbf{v}$.

\section{CENTRALIZED MULTI-CHANNEL WIENER FILTER (MWF)}

In this section, we first consider the centralized MWF, which has access to all the signals in $\mathbf{y}$. The MWF allows to estimate a hidden desired signal with an on-off characteristic, by means of a linear combination of the given set of signals [20]. We assume without loss of generality that the EEG signals are pre-processed such that they have a zero DC component, i.e., $E\{\mathbf{y}\}=\mathbf{0}$, where $E\{\cdot\}$ denotes the expected value operator. We define the network-wide covariance matrix

$$
\mathbf{R}_{y y}=E\left\{\mathbf{y y}^{T}\right\} .
$$

Since $\mathbf{d}$ and $\mathbf{v}$ can be assumed to be independent, we have that

$$
\mathbf{R}_{y y}=\mathbf{R}_{d d}+\mathbf{R}_{v v}
$$

where $\mathbf{R}_{d d}=E\left\{\mathbf{d d}^{T}\right\}$ and $\mathbf{R}_{v v}=E\left\{\mathbf{v} \mathbf{v}^{T}\right\}$. To compute the optimal linear spatial filter to estimate the eye blink artifacts $d_{j}$ in the $j$-th EEG channel $y_{j}$ in a minimum mean square error (MMSE) sense, we have to solve for

$$
\min _{\mathbf{w}} E\left\{\left(d_{j}-\mathbf{w}^{T} \mathbf{y}\right)^{2}\right\}
$$

which is equivalent to solving

$$
\min _{\mathbf{w}} \mathbf{w}^{T} \mathbf{R}_{y y} \mathbf{w}-2 \mathbf{w}^{T} \mathbf{r}_{y d_{j}}
$$

where $\mathbf{r}_{y d_{j}}=E\left\{\mathbf{y} \cdot d_{j}\right\}$. The solution of (5) is [20,26]

$$
\hat{\mathbf{w}}(j)=\mathbf{R}_{y y}^{-1} \mathbf{r}_{y d_{j}} .
$$

In the sequel, the hat-notation refers to the centralized MWF solution, and the argument $(j)$ refers to the fact that it extracts the eye blink artifact in the $j$-th channel. Due to the independence between $\mathbf{d}$ and $\mathbf{v}$, we have that $\mathbf{r}_{y d_{j}}=E\left\{\mathbf{y} \cdot d_{j}\right\}=E\left\{\mathbf{d} \cdot d_{j}\right\}=\mathbf{R}_{d d} \mathbf{e}_{j}$, where $\mathbf{e}_{j}$ is a vector that selects the $j$-th column of $\mathbf{R}_{d d}$, i.e., it is an all-zero vector, except for the $j$-th entry, which is equal to 1 . Therefore, the MWF solution (6) can be written as

$$
\hat{\mathbf{w}}(j)=\mathbf{R}_{y y}^{-1} \mathbf{R}_{d d} \mathbf{e}_{j} .
$$

While $\mathbf{R}_{y y}$ can be estimated based on a temporal averaging, the direct estimation of $\mathbf{R}_{d d}$ is not possible, since $\mathbf{d}$ is not an observed signal. However, using (3), we can rewrite (7) as

$$
\hat{\mathbf{w}}(j)=\left(\mathbf{I}-\mathbf{R}_{y y}^{-1} \mathbf{R}_{v v}\right) \mathbf{e}_{j}
$$

where $\mathbf{I}$ denotes the identity matrix. $\mathbf{R}_{v v}$ can be estimated in signal segments which do not contain an eye blink artifact, while $\mathbf{R}_{y y}$ can be estimated in signal segments that do contain an eye blink artifact. Note that this requires an additional eye blink artifact detection algorithm, which can be based on a simple thresholding procedure, or on more advanced eye blink artifact detection algorithms described in literature [27].

Finally, to remove the eye blink artifacts from channel $y_{j}$, we 
compute

$$
\hat{v}_{j}=y_{j}-\hat{\mathbf{w}}(j)^{T} \mathbf{y} .
$$

It is noted that, if $\mathbf{e}_{j}$ is removed in (8), we obtain a matrix $\hat{\mathbf{W}}=$ $\left(\mathbf{I}-\mathbf{R}_{y y}^{-1} \mathbf{R}_{v v}\right)$ where each column represents a different spatial filter to remove the eye blink artifacts in each and every channel of $\mathbf{y}$, i.e.,

$$
\hat{\mathbf{v}}=\mathbf{y}-\hat{\mathbf{W}}^{T} \mathbf{y}=\mathbf{R}_{y y}^{-1} \mathbf{R}_{v v} \mathbf{y} .
$$

From the righthand side of (10), we see that the columns of the matrix $\mathbf{R}_{y y}^{-1} \mathbf{R}_{v v}$ contain the spatial filters that directly estimate the artifact-free EEG signals, which could have been obtained immediately from (4)-(7) by replacing $d_{j}$ with $\mathbf{v}$, and using a square matrix $\mathbf{W}$ as the optimization variable. However, we have described the EBAR algorithm as a two-step approach (estimation of the eye blink artifacts, followed by a substraction), to explicitly relate the estimation problem to the data model that is typically assumed in the DANSE algorithm (see next section), where the spatial correlation matrix of the target signal component (in this case $\mathbf{R}_{d d}$ ) is assumed to have low rank.

Finally, it is noted that, since the eye blink artifacts in each EEG channel are a scaled version of the same source signal [28], it holds that

$$
\forall i, j \in\{1, \ldots, M\}, \exists \alpha_{i j} \neq 0: d_{i}=\alpha_{i j} d_{j}
$$

i.e., the eye blink artifacts are the same in each EEG channel up to a scaling. Therefore, the optimal solution (6) theoretically yields the same spatial filter for all channels up to a scaling, i.e.,

$$
\forall i, j \in\{1, \ldots, M\}, \exists \alpha_{i j} \neq 0: \quad \hat{\mathbf{w}}(i)=\alpha_{i j} \hat{\mathbf{w}}(j) .
$$

\section{DISTRIBUTED MWF-BASED EYE BLINK ARTIFACT REMOVAL USING THE DANSE ALGORITHM}

In order to compute the optimal spatial filter (8), we have to compute the inverse of the network-wide covariance matrix $\mathbf{R}_{y y}$. At first sight, this seems to hamper a distributed implementation of the optimal $M$-channel MWF, since the estimation of $\mathbf{R}_{y y}$, as well as computing its inverse, inherently requires data centralization. In this section, we propose a distributed EBAR algorithm where each node of the WESN aims to remove the eye blink artifacts in each of its own EEG channels, based on the so-called distributed adaptive node-specific signal estimation (DANSE) algorithm [23], which can be viewed as a distributed implementation of the MWF. The term 'node-specific' refers to the fact that each node estimates a different signal, in this case the eye blink artifacts in its local EEG signal(s).

\subsection{DANSE algorithm}

The idea behind DANSE is to optimally fuse the $M_{k}$-channel signal $\mathbf{y}_{k}$ in each node into a single-channel signal $z_{k}$ with a linear fusion rule

$$
z_{k}=\mathbf{f}_{k}^{T} \mathbf{y}_{k}
$$

where the $M_{k}$-dimensional fusion vector $\mathbf{f}_{k}$ takes a specific form, as we will explain later (see (19)). The signal $z_{k}$ is then broadcast to all the other nodes in the WESN. This means that a node $k \in \mathcal{K}$ has access to $M_{k}+K-1$ input signals, i.e., its own $M_{k}$-channel signal $\mathbf{y}_{k}$, and the $K-1$ broadcast signals from the other nodes in $\mathcal{K} \backslash\{k\}$, which we stack in the vector $\mathbf{z}_{-k}=\left[\begin{array}{llllll}z_{1} & \ldots & z_{k-1} & z_{k+1} & \ldots & z_{K}\end{array}\right]^{T}$, where the ' $-k$ ' subscript refers to the fact that $z_{k}$ is not included. The $\left(M_{k}+K-1\right)$-channel input signal $\tilde{\mathbf{y}}_{k}$ at node $k$ is then defined as

$$
\tilde{\mathbf{y}}_{k}=\left[\begin{array}{c}
\mathbf{y}_{k} \\
\mathbf{z}_{-k}
\end{array}\right]=\tilde{\mathbf{d}}_{k}+\tilde{\mathbf{v}}_{k}
$$

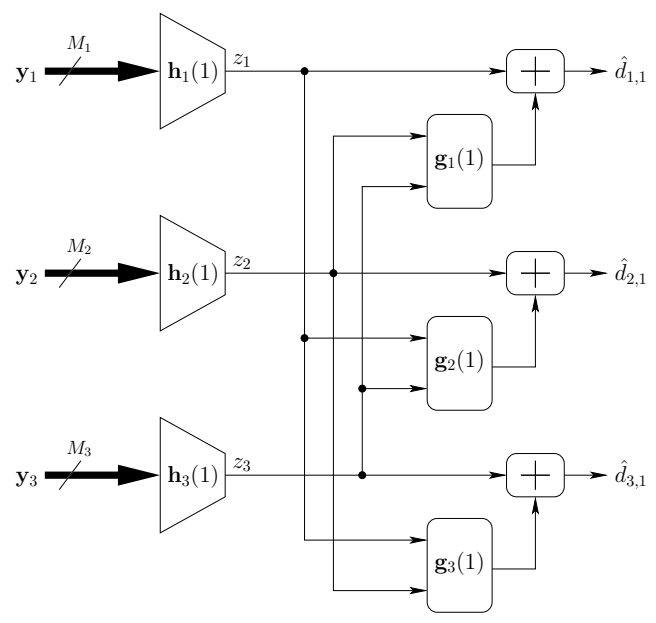

Fig. 2. Schematic representation of the DANSE algorithm in a 3node WESN, estimating the eye blink artifacts in the first channel of each node.

where $\tilde{\mathbf{d}}_{k}$ denotes the eye blink artifacts and $\tilde{\mathbf{v}}_{k}$ the artifact-free EEG signal components. Node $k$ can use the input signal $\tilde{\mathbf{y}}_{k}$ to compute a local MWF to estimate the eye blink artifacts in its local EEG channels. For the sake of an easy exposition, let us first assume that we only want to estimate the eye blink artifacts in the first channel of each node $k \in \mathcal{K}$, using the local spatial filter $\tilde{\mathbf{w}}_{k}(1)$ (compare with (8))

$$
\tilde{\mathbf{w}}_{k}(1)=\left(\mathbf{I}-\mathbf{R}_{\tilde{y}_{k} \tilde{y}_{k}}^{-1} \mathbf{R}_{\tilde{v}_{k} \tilde{v}_{k}}\right) \mathbf{e}_{1}
$$

where $\mathbf{R}_{\tilde{y}_{k} \tilde{y}_{k}}=E\left\{\tilde{\mathbf{y}}_{k} \tilde{\mathbf{y}}_{k}^{T}\right\}$ and $\mathbf{R}_{\tilde{v}_{k} \tilde{v}_{k}}=E\left\{\tilde{\mathbf{v}}_{k} \tilde{\mathbf{v}}_{k}^{T}\right\}$ can be estimated from the signal segments with and without eye blink artifacts, respectively (the nodes with highly pronounced eye blink artifacts in their own EEG channels can share their eye blink detection decisions to help other nodes). The artifact in the first EEG channel of node $k$ is then eliminated by computing (compare with (9))

$$
\hat{v}_{k, 1}=y_{k, 1}-\tilde{\mathbf{w}}_{k}(1)^{T} \tilde{\mathbf{y}}_{k} .
$$

We partition $\tilde{\mathbf{w}}_{k}(1)$ in two parts, i.e., the part applied to $\mathbf{y}_{k}$ and the part applied to $\mathbf{z}_{-k}$ :

$$
\tilde{\mathbf{w}}_{k}(1)=\left[\begin{array}{c}
\mathbf{h}_{k}(1) \\
\mathbf{g}_{k}(1)
\end{array}\right]
$$

such that (16) can be written as

$$
\hat{v}_{k, 1}=y_{k, 1}-\mathbf{h}_{k}(1)^{T} \mathbf{y}_{k}-\mathbf{g}_{k}(1)^{T} \mathbf{z}_{-k} .
$$

The DANSE algorithm then uses the vector $\mathbf{h}_{k}(1)$ as the fusion vector $\mathbf{f}_{k}$ in (13):

$$
\forall k \in \mathcal{K}: \mathbf{f}_{k}=\mathbf{h}_{k}(1) .
$$

Note that the fusion vector $\mathbf{f}_{k}$ serves both as a part of the MWF for channel 1 in node $k$ and as a fusion vector to generate $z_{k}$. This is schematically depicted in Fig. 2 for a 3-node WESN.

However, the $\mathbf{f}_{k}$ 's that generate the $z_{k}$ signals, $\forall k \in \mathcal{K}$, are now only implicitly defined, since (19) relies on the computation of (15)(17), which in turn require the fused $z_{k}$ signals from the other nodes, resulting in a chicken-and-egg problem. Therefore, the DANSE algorithm is first initialized with random entries for the $\mathbf{f}_{k}$ 's, $\forall k \in \mathcal{K}$. In subsequent iterations, the nodes will adapt their $\tilde{\mathbf{w}}_{k}(1)$ 's and $\mathbf{f}_{k}$ 's, 
$\forall k \in \mathcal{K}$, according to (15)-(19), based on the most recent observations of $\tilde{\mathbf{y}}_{k}$ (note that the covariance matrix $\mathbf{R}_{\tilde{y}_{k} \tilde{y}_{k}}$ corresponding to $\tilde{\mathbf{y}}_{k}$ changes over time due to changes in the fusion vectors at other nodes). This happens in a sequential fashion (one node at a time) [23], although a small modification of the DANSE algorithm also allows the nodes to update simultaneously [24].

\subsection{Convergence and optimality}

Under assumption (11), the DANSE algorithm has been proven to converge to the optimal centralized MWF solution [23]. Therefore, the $\tilde{\mathbf{w}}_{k}(1)$ 's, $\forall k \in \mathcal{K}$ converge to a stable equilibrium setting, in which the local estimate $\hat{v}_{k, 1}$ at each node $k$ is equal to the corresponding centralized MWF-based estimate (9), i.e., as if each node had access to all the EEG channels ${ }^{3}$. Furthermore, in this optimal operation point, the eye blink artifacts can be optimally removed in all channels $y_{k, q}$ of each node $k \in \mathcal{K}, q=1, \ldots, M_{k}$, using the local input signals $\tilde{\mathbf{y}}_{k}$. This follows straightforwardly from (12), i.e., the fact that the optimal solution (6) theoretically yields the same spatial filter for all channels, up to a scaling. This means that, even though the definition of the $z_{k}$ signals in (13)-(19) is based on the $\mathbf{h}_{k}(1)$ vectors corresponding to the first channel in each node $k \in \mathcal{K}$, the estimation problem for the other channels can rely on the same $z_{k}$ signals, i.e., without transmitting any new signals.

Remark I: The DANSE algorithm reduces the communication and computational cost, at the cost of a slower tracking or adaptation speed, as the iterative scheme takes more time to converge to the optimal operation point compared to the centralized MWF.

Remark II: It is noted that, although the paper mainly focuses on WESNs, the same distributed algorithm can be used in a wired modular EEG system, e.g., to reduce the wiring complexity, to reduce the data rate over a data bus that is shared between multiple (active) electrodes [6], or to distribute the computational cost over multiple processors.

\section{SIMULATION RESULTS}

We have first applied the centralized MWF algorithm to remove the eye blink artifacts in a continuous EEG recording with 59 channels (data set 1a from the BCI competition IV [30]). The data was downsampled to $100 \mathrm{~Hz}$ and high-pass filtered to remove the DC component. The detection of the eye blink artifacts was performed on the AF3 channel (in the 10-20 system), by means of a simple (manually tuned) thresholding operation. A window of $L=200$ samples is placed around the maximum peak of each eye blink artifact (the value $L$ was manually tuned). The samples within these eye-blink artifact windows are used to estimate $\mathbf{R}_{y y}$, whereas the other samples are used to estimate $\mathbf{R}_{v v}$. Fig. 3 shows the MWF output signal (red dashed line), which is observed to be a good estimate of the eye blink artifacts. The lower plot shows the cleaned-up EEG signal $\hat{v}_{j}$, where the eye blink artifact estimate $\hat{\mathbf{w}}(j)^{T} \mathbf{y}$ is subtracted from $y_{j}$.

In order not to corrupt the EEG signal, the component $\hat{\mathbf{w}}(j)^{T} \mathbf{v}$ should be as small as possible. Therefore, and due to lack of a ground truth, we propose the following signal-to-error ratio (SER) performance measure (incorporating all channels):

$$
S E R=10 \log _{10} \frac{\sum_{j=1}^{M} E\left\{\left(v_{j}\right)^{2}\right\}}{\sum_{j=1}^{M} E\left\{\left(\hat{\mathbf{w}}(j)^{T} \mathbf{v}\right)^{2}\right\}} .
$$

\footnotetext{
${ }^{3}$ It is noted that, even if $\alpha_{i j}=0$ in (11) for some of the channels, convergence can still be obtained with only a small modification to the DANSE algorithm [29].
}
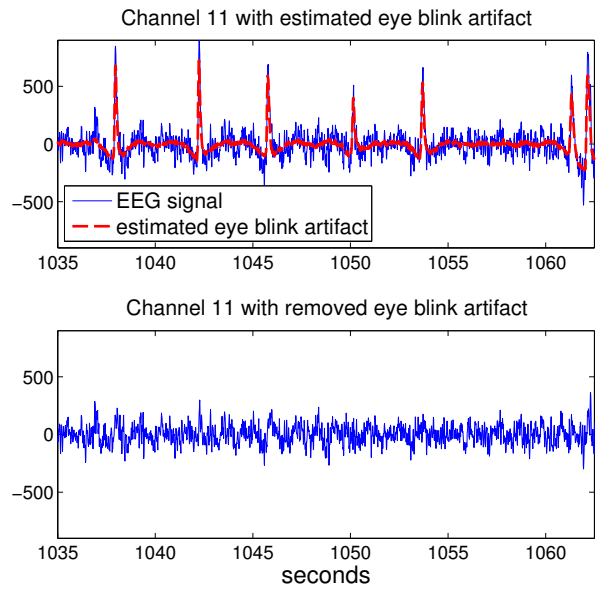

Fig. 3. Estimation of the eye blink artifact with MWF and DANSE (no observable difference).

Both the numerator and the denominator can be estimated during segments without eye blink artifacts. Using this SER measure, it is found that the MWF-based EBAR algorithm slightly outperforms the ICA-based ${ }^{4}$ Infomax algorithm in EEGLAB when applied to the EBAR problem [28] (19.9 dB for MWF versus $15.3 \mathrm{~dB}$ for ICA).

To experimentally validate the theoretical statement that the output of the DANSE algorithm is equivalent to the output of the centralized MWF, we have applied the DANSE-based EBAR algorithm to the same EEG recording. The 59 channels were divided over 6 nodes ( 10 channels per node, and 9 channels in the sixth node). The fusion vectors and the local MWFs were updated after every incoming block of 6000 samples (to capture sufficient eye blink artifacts per update). After convergence, the algorithm achieved an SER of $18.8 \mathrm{~dB}$, versus $19.9 \mathrm{~dB}$ in the centralized case. This $1 \mathrm{~dB}$ difference is negligible and not observable in Fig. 3, which therefore only shows the centralized estimate. The small discrepancy is due to the fact that (11) is only approximately satisfied, and due to unavoidable estimation errors ${ }^{5}$ in the local covariance matrices used in (15).

\section{CONCLUSIONS}

We have presented a distributed algorithm for EBAR in modular EEG systems such as WESNs. We have first presented a centralized EBAR algorithm based on the MWF, and we have explained how the DANSE algorithm allows to implement this MWF-based EBAR algorithm in a distributed fashion, resulting in a reduced computational complexity and communication cost, at the cost of a slower adaptation speed. Simulation results have validated the theoretical claim that this distributed algorithm achieves the same accuracy as the centralized MWF.

\section{REFERENCES}

[1] A. Casson, D. Yates, S. Smith, J. Duncan, and E. RodriguezVillegas, "Wearable electroencephalography," IEEE Engineering in Medicine and Biology Magazine, vol. 29, no. 3, pp. 44$56,2010$.

\footnotetext{
${ }^{4}$ The selection of the independent component corresponding to the eye blink artifacts was done manually based on a visual inspection.

${ }^{5}$ These estimation errors are neglected in the convergence proof of DANSE, where infinite observation windows are assumed.
} 
[2] A. Casson, S. Smith, J. Duncan, and E. Rodriguez-Villegas, "Wearable EEG: what is it, why is it needed and what does it entail?" in International Conference of the IEEE Engineering in Medicine and Biology Society (EMBS), 2008, pp. 58675870 .

[3] C.-T. Lin, L.-W. Ko, J.-C. Chiou, J.-R. Duann, R.-S. Huang, S.-F. Liang, T.-W. Chiu, and T.-P. Jung, "Noninvasive neural prostheses using mobile and wireless EEG," Proceedings of the IEEE, vol. 96, no. 7, pp. 1167-1183, 2008.

[4] Y. Chi, S. Deiss, and G. Cauwenberghs, "Non-contact low power EEG/ECG electrode for high density wearable biopotential sensor networks," in International Workshop on Wearable and Implantable Body Sensor Networks, 2009, pp. 246-250.

[5] G. Gargiulo, P. Bifulco, R. Calvo, M. Cesarelli, C. Jin, and A. van Schaik, "A mobile EEG system with dry electrodes," in IEEE Biomedical Circuits and Systems Conference (BioCAS), 2008, pp. 273-276.

[6] S. Patki, B. Grundlehner, A. Verwegen, S. Mitra, J. Xu, A. Matsumoto, R. Yazicioglu, and J. Penders, "Wireless EEG system with real time impedance monitoring and active electrodes," in IEEE Biomedical Circuits and Systems Conference (BioCAS), 2012, pp. 108-111.

[7] M.-F. Wu and C.-Y. Wen, "Distributed cooperative sensing scheme for wireless sleep EEG measurement," IEEE Sensors Journal, vol. 12, no. 6, pp. 2035-2047, 2012.

[8] _ , "The design of wireless sleep EEG measurement system with asynchronous pervasive sensing," in Systems, Man and Cybernetics, 2009. SMC 2009. IEEE International Conference on, 2009, pp. 714-721.

[9] B. Latré, B. Braem, I. Moerman, C. Blondia, and P. Demeester, "A survey on wireless body area networks," Wireless Networks, vol. 17, no. 1, pp. 1-18, Jan. 2011.

[10] H. Cao, V. Leung, C. Chow, and H. Chan, "Enabling technologies for wireless body area networks: A survey and outlook," IEEE Communications Magazine, vol. 47, no. 12, pp. 84-93, 2009.

[11] M. Sun, W. Jia, W. Liang, and R. Sclabassi, "A low-impedance, skin-grabbing, and gel-free EEG electrode," in International Conference of the IEEE Engineering in Medicine and Biology Society (EMBC), 2012, pp. 1992-1995.

[12] T. Someya, "Building bionic skin," IEEE Spectrum, vol. 50, no. 9, pp. 44-49, 2013.

[13] J. R. Ives, "New chronic EEG electrode for critical/intensive care unit monitoring," Journal of Clinical Neurophysiology, vol. 22, no. 2, pp. 119-123, April.

[14] A. Casson and E. Rodriguez-Villegas, "Data reduction techniques to facilitate wireless and long term AEEG epilepsy monitoring," in Neural Engineering, 2007. CNE '07. 3rd International IEEE/EMBS Conference on, 2007, pp. 298-301.

[15] N. Verma, A. Shoeb, J. V. Guttag, and A. Chandrakasan, "A micro-power EEG acquisition soc with integrated seizure detection processor for continuous patient monitoring," in Symposium on VLSI Circuits, 2009, pp. 62-63.

[16] S. Makeig, A. J. Bell, T.-p. Jung, and T. J. Sejnowski, "Independent component analysis of electroencephalographic data," Advances in Neural Information Processing Systems, vol. 8, pp. 145-151, 1996.
[17] Y. Li, Z. Ma, W. Lu, and Y. Li, "Automatic removal of the eye blink artifact from EEG using an ica-based template matching approach," Physiological Measurement, vol. 27, no. 4, p. 425, 2006.

[18] J. Xie, T. Qiu, and L. Wen-hong, "An ocular artifacts removal method based on canonical correlation analysis and two-channel EEG recordings," in World Congress on Medical Physics and Biomedical Engineering May 26-31, 2012, Beijing, China, ser. IFMBE Proceedings, M. Long, Ed. Springer Berlin Heidelberg, 2013, vol. 39, pp. 465-468.

[19] S. Casarotto, A. M. Bianchi, S. Cerutti, and G. A. Chiarenza, "Principal component analysis for reduction of ocular artefacts in event-related potentials of normal and dyslexic children," Clinical Neurophysiology, vol. 115, no. 3, pp. 609-619, 2004.

[20] S. Doclo and M. Moonen, "GSVD-based optimal filtering for single and multimicrophone speech enhancement," IEEE Transactions on Signal Processing, vol. 50, no. 9, pp. 2230 2244, Sep. 2002.

[21] B. Van Dun, J. Wouters, and M. Moonen, "Multi-channel Wiener filtering based auditory steady-state response detection," in IEEE International Conference on Acoustics, Speech and Signal Processing (ICASSP), vol. 2, 2007, pp. II-929-II932.

[22] A. Bertrand, V. Mihajlovic, B. Grundlehner, C. Van Hoof, and M. Moonen, "Motion artifact reduction in EEG recordings using multi-channel contact impedance measurements," in Proc. IEEE Biomedical Circuits and Systems Conference (BioCAS), Rotterdam, The Netherlands, Nov. 2013.

[23] A. Bertrand and M. Moonen, "Distributed adaptive nodespecific signal estimation in fully connected sensor networks - part I: sequential node updating," IEEE Transactions on Signal Processing, vol. 58, pp. 5277-5291, 2010.

[24] — - "Distributed adaptive node-specific signal estimation in fully connected sensor networks - part II: simultaneous \& asynchronous node updating," IEEE Transactions on Signal Processing, vol. 58, pp. 5292-5306, 2010.

[25] — - "Distributed adaptive estimation of node-specific signals in wireless sensor networks with a tree topology," IEEE Trans. Signal Processing, vol. 59, no. 5, pp. 2196-2210, May 2011.

[26] S. Haykin, Adaptive Filter Theory, 3rd ed. Prentice Hall, 1996.

[27] Z. Tiganj, M. Mboup, C. Pouzat, and L. Belkoura, "An algebraic method for eye blink artifacts detection in single channel EEG recordings," in Int. Conf. on Biomagnetism (Biomag2010), ser. IFMBE Proceedings, S. Supek and A. Suac, Eds. Springer Berlin Heidelberg, 2010, vol. 28, pp. $175-178$.

[28] A. Delorme and S. Makeig, "EEGLAB: an open source toolbox for analysis of single-trial EEG dynamics including independent component analysis," Journal of Neuroscience Methods, vol. 134, no. 1, pp. 9 - 21, 2004.

[29] A. Bertrand and M. Moonen, "Robust distributed noise reduction in hearing aids with external acoustic sensor nodes," EURASIP Journal on Advances in Signal Processing, vol. 2009, Article ID 530435, 14 pages, 2009. doi:10.1155/2009/530435.

[30] M. Tangermann et al., "Review of the BCI competition IV," Frontiers in Neuroscience, vol. 6, no. 55, 2012. 